\title{
Haemolytic disease of the newborn
}

\author{
Neil A Murray, Irene A G Roberts
} The changing management of haemolytic disease of the newborn is
reviewed

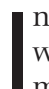

the space of most paediatricians working lifetime, the spectrum of haemolytic disease of the newborn (HDN) has changed beyond recognition. Thirty years ago, HDN was almost synonymous with Rh D allo-immunisation and was a common neonatal problem. It was usually unmodified by antenatal therapy and caused overt fetal or neonatal haemolysis, leading to marked neonatal hyperbilirubinaemia and attendant anaemia. Affected neonates were commonly sick and unstable at birth, required multiple exchange transfusions, and suffered considerable neonatal morbidity and mortality. However, the introduction in the UK in the 1970s of routine postnatal prophylactic anti-D immunoglobulin for Rh $\mathrm{D}$ negative women has dramatically reduced this form of HDN, and as such this treatment ranks as one of the great success stories of modern perinatal care.

Although the clinical burden imposed on neonatal services by HDN has greatly reduced, it has not disappeared. Neonatal paediatricians continue to recognise a number of different presentations of neonatal haemolysis, many of which are considerably more subtle than the traditional "neonatal emergency" presentation of severe $\mathrm{Rh} \mathrm{D}$ disease. In contemporary neonatal practice, HDN should be considered in the fetus or neonate where there is one or more of the following:

- rapidly developing or severe hyperbilirubinaemia not predicted by maternal antenatal antibody screening;

- positive maternal antenatal antibody screening and/or diagnosis of a severely anaemic/hydropic fetus;

- a positive direct anti-globulin test (DAT);

- haemolysis detected on blood film examination;

- prolonged hyperbilirubinaemia.

In addition to these various presentations of HDN, the spectrum of the disease has altered as new treatments, particularly improved phototherapy and/or intravenous immunoglobulin (IVIG), are being evaluated and introduced to reduce the need for exchange transfusion in the reduced number of severe cases. This review will aim to place $\mathrm{HDN}$ in the context of contemporary neonatal services, systematically evaluate its various presentations, and review the evolving role of these new treatments.

\section{UNEXPECTED RAPIDLY DEVELOPING OR SEVERE HYPERBILIRUBINAEMIA}

This is now the clinical presentation that most often alerts neonatal paediatricians to newborns with HDN. All will be familiar with the relatively well term neonate presenting within the first $48 \mathrm{~h}$ of life with either early-onset jaundice or a total serum bilirubin that has already exceeded $300 \mu \mathrm{mol} / \mathrm{l}$. By implication, the problem in such neonates has not been predicted by maternal antenatal antibody screening, as appropriate investigation, monitoring procedures and treatment would already have been started. Table 1 gives the most common causes of HDN presenting in this way.

\section{ABO INCOMPATIBILITY}

ABO HDN occurs almost exclusively in the offspring of women of blood group O, although reports exist of occasional cases in group A mothers with high-titre anti(group B) IgG. ${ }^{1}$ In general, $15-25 \%$ of all maternal/fetal pairs are ABO-incompatible, but $\mathrm{ABO} \mathrm{HDN}$ is confined to the roughly $1 \%$ of such group $\mathrm{O}$ women who have antenatal high-titre IgG antibodies. ${ }^{2}$ Haemolysis due to anti- A is more common ( 1 in 150 births) than that to anti- B. Affected neonates will usually be DAT positive, but, in contrast with the clinical picture with anti-Rh antibodies, both anti-A and anti-B HDN usually result predominantly in hyperbilirubinaemia without significant neonatal anaemia. This is mainly because of the relatively few group A or B antigenic sites on neonatal red blood cells (RBCs), allowing the antibody-coated cells to remain in the circulation for a longer period than in $\mathrm{Rh} \mathrm{D}$ disease. ${ }^{3}$ As a reflection of this, the blood film in $\mathrm{ABO}$ haemolytic disease characteristically shows very large numbers of spherocytes, with little or no increase in nucleated RBCs (nRBCs), whereas in Rh D HDN there are few spherocytes and large numbers of circulating nRBCs. Management of ABO HDN is usually successful with phototherapy alone provided by modern equipment (see below). However, close monitoring of the affected neonate is essential, and exchange transfusion is occasionally required. This is particularly the case in $\mathrm{ABO} \mathrm{HDN}$ due to anti-B IgG where racial differences in disease severity exist, severe cases being prevalent in mothers and neonates of black African origin. In such cases, severe anaemia as well as hyperbilirubinaemia ${ }^{4}$ can occur and rarely antenatal hydrops fetalis has been described. ${ }^{5}$

\section{RBC MEMBRANE DISORDERS}

The main clues that a neonate with earlyonset or rapidly progressive hyperbilirubinaemia has a RBC membrane disorder are a family history, non-immune (DAT negative) haemolysis and an abnormal blood film. Indeed, neonatal RBC membrane disorders can nearly always be recognised by the characteristic shape of RBCs on a blood film. However, the identification of the exact type of membrane abnormality is more complex and requires specialised investigations on both the neonate and the parents and close liaison with a haematologist. RBC membrane electrophoresis is the definitive investigation if the clinical pattern and appearance of the family blood films suggest a membrane disorder, as the osmotic fragility test is of limited value in neonates. It is essential to carry out these diagnostic investigations (RBC membrane studies) on pre-transfusion blood samples to minimise diagnostic confusion resulting from transfused cells. ${ }^{6}$

Table 1 Causes of haemolytic disease of the newborn presenting as early-onset or rapidly progressive hyperbilirubinaemia not predicted by maternal antibody screening

$\mathrm{ABO}$ incompatibility (most common combination: maternal group $\mathrm{O} /$ neonate group $\mathrm{A}$ Red blood cell membrane defects (eg hereditary spherocytosis)

Red blood cell enzyme defects (eg glucose-6-phosphate dehydrogenase (G6PD) deficiency) Thalassaemia (rarely $\alpha$-thalassaemia major in preterm neonates) 


\section{Hereditary spherocytosis}

This is the most common RBC membrane defect. It occurs in 1 in 5000 live births to parents of Northern European extraction, but is less often seen in other ethnic groups. ${ }^{7}$ The usual presentation of hereditary spherocytosis in the neonate is with unconjugated hyperbilirubinaemia. Most affected neonates are not anaemic, but a small number develop anaemia severe enough to require transfusion. Hereditary spherocytosis is inherited as an autosomal dominant trait, but around $25 \%$ of cases occur as the result of new mutations. It is genetically heterogeneous; mutations in spectrin, ankyrin, protein 4.1 and protein 3 have all been reported. ${ }^{78}$ The blood film shows moderate numbers of spherocytes with an appearance identical with that of $\mathrm{ABO}$ HDN. However, (as above) the two disorders are distinguishable by the negative DAT in hereditary spherocytosis. Whereas some babies will require one or two transfusions during the first $1-2$ months of life, very few remain transfusiondependent after this time. In the ongoing assessment of such neonates, it is important to pause "top-up" of a RBC transfusion programme to evaluate if steady-state clinical and haemoglobin $(\mathrm{Hb})$ concentrations have been reached. All babies with hereditary spherocytosis should receive folic acid supplementation in the first year of life $(500 \mu \mathrm{g} / \mathrm{kg} /$ day or a total weekly dose of $2.5 \mathrm{mg}$ is recommended).

\section{Hereditary elliptocytosis}

This is a more complex disorder. It is caused by different mutations in the genes for spectrin, ankyrin or protein 4.1 than those seen in hereditary spherocytosis. ${ }^{78}$ In the common, autosomal dominant form of hereditary elliptocytosis, the heterozygotes have no clinical manifestations (ie no anaemia and no jaundice) apart from elliptocytes on the blood film. No treatment is required and folic acid supplementation is not necessary. However, neonates who are homozygous or compound heterozygotes for hereditary elliptocytosis mutations have severe haemolytic anaemia; the most important form is hereditary pyropoikilocytosis (HPP).

\section{Hereditary pyropoikilocytosis}

Neonates with HPP have more than one mutation in a RBC membrane protein (they may be homozygous or compound heterozygotes)..$^{78}$ Although HPP is not common, it is clinically important, as it leads to severe, transfusion-dependent, haemolytic anaemia in affected neonates and infants. The diagnosis of HPP can be made definitively by examining blood films from the neonate that show a high proportion of bizarre fragmented RBCs and microspherocytes. A further useful diagnostic clue is the low mean corpuscular volume at birth $(<60 \mathrm{fl})$. Parental blood films normally show that one or both parents have RBC elliptocytosis. RBC transfusion is usually necessary until the child is old enough to undergo splenectomy, to which there is an excellent response.

\section{RBC ENZYME DEFECTS}

The main RBC enzyme defects that present in the neonatal period are glucose-6-phosphate dehydrogenase (G6PD) deficiency and pyruvate kinase deficiency. They usually present with early-onset unconjugated hyperbilirubinaemia and can be distinguished from RBC membrane disorders in that there are usually no diagnostic changes on the blood film and by their inheritance pattern (G6PD deficiency being $\mathrm{X}$-linked so rare in female neonates).

\section{G6PD deficiency}

G6PD deficiency is seen in all ethnic groups but has a high prevalence in people from central Africa (20\%) and the Mediterranean (10\%). In neonatal G6PD deficiency, jaundice usually presents within the first few days of life and is often severe. In contrast, anaemia is extremely rare and the blood film is completely normal. So the diagnosis must be made by assaying G6PD on a peripheral blood sample.' It is not clear why some, but not all, G6PD-deficient neonates develop neonatal jaundice. In addition, the pathogenesis of the jaundice is also unclear, as most babies with G6PD deficiency have no evidence of haemolysis. As severe hyperbilirubinaemia is common, close monitoring of the bilirubin concentration is essential, particularly when interactions with other risk factors for neonatal hyperbilirubinaemia are present, such as hereditary spherocytosis or Gilbert's syndrome, as kernicterus has been reported in this setting. ${ }^{10} 11$ Counselling for parents of neonates with G6PD deficiency about which medicines, chemicals and foods may precipitate haemolysis ${ }^{12}$ is also an essential followup activity in such families. Certain uncommon variants of G6PD deficiency are associated with chronic haemolysis, and for these children folic acid supplements should be given. ${ }^{9}$ However, for the vast majority of patients, there is no chronic haemolysis and no anaemia and therefore folic acid supplements are not indicated

\section{Pyruvate kinase deficiency}

Pyruvate kinase deficiency is the second most common RBC enzymopathy in neonates. It is autosomal recessive and clinically heterogeneous, with presentation varying from hydrops fetalis, through severe early-onset neonatal hyperbilirubinaemia requiring exchange transfusion, to a mild unconjugated hyperbilirubinaemia mimicking physiological jaundice. ${ }^{13}{ }^{14}$ The diagnosis is made by measuring pretransfusion RBC pyruvate kinase activity. In mild cases, the pyruvate kinase activity may be relatively modestly reduced, making the diagnosis difficult and it is often useful to assay levels in the parents for confirmation. The blood film is sometimes distinctive, but more often shows non-specific changes of non-spherocytic haemolysis, and therefore it is good practice to assay pyruvate kinase in all babies with unexplained haemolysis after the common causes have been excluded. After the neonatal period, some, but not all, children are transfusion-dependent, and folic acid supplements should be given to prevent deficiency due to chronic haemolysis.

\section{Other RBC enzymopathies}

The other RBC enzymopathies are rare. The most important to be aware of in the neonatal period is triosephosphate isomerase deficiency, which is autosomal recessive. ${ }^{15}$ One third of patients present with neonatal haemolytic anaemia, and this may be the only presenting feature at this age. The devastating neurological features of this disorder only become apparent 6-12 months later, ${ }^{15}$ emphasising the point that patients with persistent neonatal haemolysis should always be fully investigated and followed up appropriately.

\section{NEONATAL HAEMOLYSIS DUE TO HAEMOGLOBINOPATHIES}

The haemoglobinopathies, except for $\alpha$ thalassaemia major, do not usually present in the neonatal period. Occasional non-thalassaemic, structural $\alpha$-globin and $\gamma$-globin gene mutations, which are clinically completely silent in adults and children, cause transient haemolytic anaemia in the neonate (see below). Symptoms and signs of the major $\beta$ globin haemoglobinopathies (sickle cell disease and $\beta$-thalassaemia major) are rare in neonates, although modern techniques (eg high-performance liquid chromatography (HPLC), isoelectric focusing) allow the diagnosis to be made on neonatal blood samples where family studies indicate that both parents are carriers. ${ }^{16}$

\section{$\alpha$-Thalassaemia major}

$\alpha$-Thalassaemia major occurs when all four $\alpha$-globin genes on chromosome 16 are deleted. ${ }^{17}$ It predominantly affects 
families of south east Asian origin and presents with mid-trimester fetal anaemia or hydrops fetalis, which is fatal within hours of delivery (occasionally babies have lived a few days). It can occasionally cause diagnostic confusion if a fetus developing the marked anaemia and hydrops characteristic of the condition is delivered preterm and then rapidly develops hyperbilirubinaemia. Therefore, the diagnosis of $\alpha$-thalassaemia major should be suspected in any case of severe fetal anaemia presenting in the second trimester and any case of hydrops fetalis with severe anaemia in which the parents come from south east Asia (it is also seen occasionally in families who originate from India, the Middle East or the Mediterranean). Checking the blood counts of the parents will immediately identify whether they are at risk of having a child with $\alpha$-thalassaemia major: both parents will be carriers of a chromosome 16 in which both of the two $\alpha$-globin genes are deleted and so they will have hypochromic, microcytic RBC indices (mean corpuscular volume usually $<74$ $\mathrm{fl}$ and mean corpuscular $\mathrm{Hb}$ usually $<24 \mathrm{pg}$ ). The diagnosis of $\alpha$-thalassaemia major is confirmed by $\mathrm{Hb}$ electrophoresis or HPLC (which shows only Hb Barts and $\mathrm{Hb}$ Portland; $\mathrm{HbF}$ and $\mathrm{HbA}$ are absent). The blood film shows hypochromic, microcytic RBCs with vast numbers of circulating nRBCs. Neonatal management of $\alpha$-thalassaemia major has no impact on survival unless the baby has received a planned programme of intrauterine transfusions. For fetuses/neonates treated in this way, postnatal management is the same as for $\beta$-thalassaemia-that is, lifelong RBC transfusions or bone marrow transplantation after the age of 2 years. ${ }^{18} 19$

\section{$\alpha$-Globin and $\gamma$-globin chain structural abnormalities}

Most $\alpha$-globin and $\gamma$-globin gene variants are clinically silent. Occasional $\alpha$-globin gene variants may cause haemolytic anaemia in the newborn because, when the abnormal $\alpha$-globin associates with $\gamma$ globin, the resultant $\mathrm{Hb}$ is unstable, whereas, when the variant $\alpha$-globin associates with $\beta$-globin, the resultant $\mathrm{Hb}$ is stable. An example of this is $\mathrm{Hb}$ Hasharon: $\alpha^{214 \text { Asp- }>\text { His }}-\gamma_{2}$ is unstable, but, as $\gamma$-globin chain production is physiologically switched off and $\beta$-globin chain production predominates, the $\alpha^{214 A s p-}$

$>$ His $-\beta_{2}$ produced is stable and the haemolytic anaemia completely resolves. ${ }^{4} \mathrm{~A}$ similar principle occurs in the $\gamma$-globin variant HbF-Poole which causes neonatal haemolytic anaemia, which resolves as the switch from $\gamma$-globin to $\beta$-globin occurs. ${ }^{20}$ These variants can be identified by Hb HPLC and are worth considering when more common causes of unexplained haemolysis have been excluded.

\section{POSITIVE MATERNAL ANTIBODY SCREENING}

Although most acute presentations of neonatal hyperbilirubinaemia/haemolysis now occur without antenatal warning, there continues to be a considerable number a cases of HDN predicted by antenatal screening. The principal alloantibodies leading to these forms of HDN are those directed against $\mathrm{Rh}$ antigens (anti-D, anti-c and anti-E), anti-Kell, anti-Kidd $\left(\mathrm{J}^{\mathrm{k}}\right)$, anti-Duffy $\left(\mathrm{F}^{\mathrm{y}}\right)$ and antibodies of the MNS blood group system, including anti-U. Of these, anti-D remains the most common cause of significant haemolytic anaemia, affecting 1 in 1200 pregnancies. ${ }^{21-23}$ Anti-Kell antibodies are less common, but can cause severe fetal and neonatal anaemia as they inhibit erythropoiesis as well as causing haemolysis. ${ }^{24}$ The numbers of such cases are decreasing, but maternal/fetal pairs with these complications continue to represent a disproportionate part of the spectrum (and workload) of HDN as they require frequent antenatal monitoring and sometimes treatment-that is, repeated intrauterine RBC transfusion. Although beyond the scope of this review, important advances have been made in the monitoring of fetuses/neonates whose mothers carry significant allo-antibodies and who are therefore potential candidates for HDN. Antenatal blood group genotyping by polymerase chain reaction from fetal cells obtained by amniocentesis aids in the definitive identification of susceptible fetuses. ${ }^{25}$ In time, this may also be possible as a routine by recovering fetal cells from the maternal circulation. ${ }^{26} 27$ In addition, accurate non-invasive assessment of the degree of fetal anaemia is now possible by using serial middle cerebral artery Doppler studies, thus helping to avoid repeated invasive procedures. For a more detailed overview of these advances, the reader is referred to two reviews. ${ }^{28} 29$

\section{MANAGEMENT OF HDN}

The antenatal diagnosis and postnatal management of fetuses/neonates affected by RBC allo-immunisation continues to require close cooperation between obstetric, neonatal and haematology teams. Not withstanding the results of antenatal investigations and management, all neonates at risk should have cord blood taken for measurement of $\mathrm{Hb}, \mathrm{DAT}$, and bilirubin (to confirm diagnosis, assess the effectiveness of any antenatal treatment, and predict the likely need for postnatal treatment). All potentially affected neonates should remain in hospital until hyperbilirubinaemia and/or anaemia have been properly assessed and managed and appropriate follow-up arranged. Trial evidence is lacking to support the use of prophylactic phototherapy in affected neonates, ${ }^{30}$ but in busy postnatal wards this approach at least ensures that those who require effective phototherapy receive it in a timely manor and this may avoid exchange transfusion in a small number of neonates. Appropriate guidelines for exchange transfusion in neonates with HDN are becoming a more difficult area. Traditional guidelines suggest that exchange transfusion in HDN is required for:

- severe anaemia: $\mathrm{Hb}<\mathrm{l} 0 \mathrm{~g} / \mathrm{dl}$ at birth (with the possible exception of antiKell, which, as outlined above, can cause considerable anaemia without appreciable hyperbilirubinaemia); and/ or

- severe hyperbilirubinaemia (total serum bilirubin $>350 \mu \mathrm{mol} / \mathrm{l}$ ) in the first $48 \mathrm{~h}$ of life or rapidly increasing hyperbilirubinaemia (total serum bilirubin increasing $>10 \mu \mathrm{mol} / \mathrm{l}$ per hour).

However, these values are derived from clinical experience in preventing the development of kernicterus in sick neonates with untreated Rh D HDN (a progressively reducing clinical entity). As outlined above, the "typical" presentation of HDN is now the well term neonate with $\mathrm{ABO}$ incompatibility who presents with a total serum bilirubin already in excess of $300 \mu \mathrm{mol} / \mathrm{l}$ (sometimes as a readmission from home) without clinically significant anaemia. Intensive phototherapy during the 4-6 h it normally takes to organise an exchange transfusion almost always results in a considerable fall in the total serum bilirubin and thereby almost always removes the clinical indication for exchange transfusion. This represents a modern clinical dilemma in neonatal practice, as blood products used for exchange transfusion in the UK are derived from highly restricted donors, they are in short supply, and potential wastage of these products represents an important issue for both the National Blood Service and neonatal paediatricians. Therefore, although the guidelines for exchange transfusion outlined above remain pertinent for untreated HDN associated with defined antenatally detected maternal antibodies, most cases must now be assessed on their individual merits, with particular attention paid to the general condition of the neonate (state of hydration, gestational and postnatal age), family history, rapid achievement of an accurate clinical/laboratory diagnosis, and initial response to modern 
intensive phototherapy (see below). Following this approach, most large maternity/neonatal services now perform only a handful of exchange transfusions per year without apparent detriment to the long-term health of neonates affected by HDN. ${ }^{31}$

\section{NEONATES TREATED WITH INTRAUTERINE RBC TRANSFUSIONS}

Neonates with haemolytic disease who have received repeated intrauterine transfusions have a significantly altered neonatal course, as their fetal therapy substantially alters the underlying haemolytic disease process. The effect on fetal $\mathrm{Hb}$ of the first intrauterine transfusion is shortest-lived because of the continued presence of residual antibodycoated fetal RBCs. However, with subsequent intrauterine transfusions, fetal haemopoiesis is suppressed, and the fetal circulation is then maintained by compatible transfused RBCs that are not susceptible to the haemolytic process. As a result, in newborns that have been treated with a successful intrauterine transfusion programme until near term, neither neonatal jaundice nor immediate anaemia are major problems and few require postnatal exchange transfusion. ${ }^{32}$ In contrast, late anaemia in such neonates is common, as the haemopoietic suppression induced by the intrauterine transfusion programme may last for many weeks after delivery. Management of such neonates is expectant, initially ensuring adequate nutritional support (including appropriate iron and vitamin supplementation). After discharge, monitoring of $\mathrm{Hb}$ concentration, blood film morphology, reticulocyte count and bilirubin is necessary to gauge when haemolysis has stopped and endogenous RBC production has re-commenced. The appropriate interval for this must be tailored to the individual neonate, taking into consideration the activity of the underlying haemolytic process and, most importantly, the general condition and growth and development of the affected neonate (simply bleeding such neonates weekly often only raises maternal anxiety without necessarily adding useful clinical information). RBC transfusions are only required in those with symptomatic anaemia (notoriously difficult to evaluate clinically $y^{33}$ ), with most neonates requiring no RBC top-ups, although in some clinically well neonates the $\mathrm{Hb}$ concentration may fall as low as $5 \mathrm{~g} / \mathrm{dl}$ before rising spontaneously (personal experience).

\section{POSITIVE DAT}

The DAT (Coombs test) is one of the cornerstones of diagnosis of HDN. It is a screening test for non-agglutinating antibodies present on an individual's RBCs. If maternal serum contains an IgG class, immunoglobulin directed against a fetal RBC antigen transplacental passage of this antibody will result in RBC antibody coating and a positive fetal/neonatal DAT. However, it should be appreciated that as a basic screening test, it has poor positive predictive value in identifying neonates who will require treatment of HDN by phototherapy. In a recent publication, Dinesh $^{34}$ reported that only $23 \%$ of neonates found to have a positive DAT on neonatal screening actually went on to develop hyperbilirubinaemia requiring phototherapy. Indeed it was only when the DAT was strongly positive $(4+)$ that $100 \%$ of such neonates required phototherapy. This study also confirmed the preponderance of $\mathrm{ABO}$ incompatibility as the main cause of HDN in modern neonatal practice, with $87 / 93$ cases of DAT positivity occurring in ABO-incompatible mother/neonate pairs. In addition, 21/145 neonates requiring neonatal phototherapy were both DAT positive and from ABO-incompatible mother/neonate pairs, with a further $17 / 145$ neonates requiring neonatal phototherapy being DAT negative but also from ABO-incompatible mother/neonate pairs.

It must also be appreciated that, in a unit where $\mathrm{Rh} \mathrm{D}$ negative women receive routine antenatal anti-D immunoglobulin in the third trimester, up to $15 \%$ of the resulting neonates will have a positive DAT simply from passive transfer of the prophylactic anti-D administered to the mother during pregnancy. ${ }^{35}$ These antibodies do not induce fetal or neonatal haemolysis and so no special postnatal investigation or monitoring of such neonates is required (although it is all too easy for such neonates to be included in follow-up programmes for HDN). If doubt exists as to the cause of the DAT positivity in such neonates, blood film examination, by an appropriately experienced person, will give the correct diagnosis, as spherocytes, nRBCs and RBC fragments will not be present in unusual numbers.

\section{HAEMOLYSIS DETECTED ON BLOOD FILM EXAMINATION}

As the recognised presentations of HDN become more varied and the number, and complexity, of identified causes increases, it becomes even more imperative for neonatal paediatricians to develop close working relationships with their local haematologists. In many cases, the first clinician who suspects/diagnoses HDN in a particular neonate will be the haematologist after a blood film examination for other reasons-for example, review for evidence of sepsis. This fact simply emphasises the importance of requesting blood film examination routinely on all neonates with non-physiological jaundice (table 1) and the importance of encouraging haematologists to develop skills appropriate to neonatal medicine.

\section{PROLONGED \\ HYPERBILIRUBINAEMIA}

All neonates with prolonged hyperbilirubinaemia should be thoroughly investigated to exclude the possibility of HDN (table 1). Persistent jaundice is a common presentation of HDN, and virtually all the conditions mentioned in this review may present with prolonged hyperbilirubinaemia. Achieving the correct diagnosis may well have implications not only for the jaundiced neonate but also for other family members.

\section{DEVELOPMENTS IN TREATMENT FOR NEONATAL HYPERBILIRUBINAEMIA Improved phototherapy}

The changing clinical practice surrounding HDN is, in no small way, the result of improvements in both the understanding and delivery of phototherapy. Phototherapy was first introduced for the treatment of neonatal hyperbilirubinaemia in the late $1950 \mathrm{~s},{ }^{36}$ when white light was the mainstay of treatment. Since then, considerable advances have been made, and it is now appreciated more fully that the efficacy of phototherapy in reducing neonatal hyperbilirubinaemia is dependent on a number of factors: $:^{37}$

- spectral qualities of the delivered light (optimal wavelength range 400 $520 \mathrm{~nm}$, with peak emissions of $460 \mathrm{~nm})$

- irradiance (intensity of light) ${ }^{38}$;

- surface area receiving phototherapy;

- skin pigmentation;

- total serum bilirubin concentration at the start of phototherapy;

- duration of exposure.

Thus modern phototherapy devices are designed to maximise the efficacy of phototherapy to the neonate, and clinicians are more appreciative of the importance of ensuring that such devices are used correctly-that is, ensuring the correct distance between the device and patient, and ensuring proper maintenance and servicing of phototherapy units. As a result of this, phototherapy units have become smaller in size, more easy to use around a neonate's cot, more efficient, particularly high-intensity gallium nitride light-emitting diodes, ${ }^{39} 40$ and the total irradiance that can be applied to an individual neonate has vastly 
increased. In short, phototherapy is now a viable alternative to the planned use of exchange transfusion in the treatment of even moderate to severe HDN, ${ }^{41}$ and, as devices continue to develop and improve, phototherapy will play an even greater role in the treatment of HDN. For a fuller description of developments in neonatal phototherapy since its first use, the reader is referred to two reviews. ${ }^{37} 42$

\section{High-dose IVIG}

In the last $10-15$ years, a number of pilot studies have been published reporting the effect of high-dose IVIG as an adjuvant treatment to standard therapy for HDN. ${ }^{43-}$ ${ }^{48}$ Most of these studies have also formed the basis of two systematic reviews. ${ }^{49} 50$ Clearly, in the context of their applicability to modern neonatal practice, there are potential methodological difficulties with most of these studies: the studies have assessed a relatively small number of neonates; most patients studied have ABO HDN (and are therefore presumably clinically well); the protocols of the larger studies include exchange transfusion at relatively low concentrations of bilirubin, 290-340 $\mu \mathrm{mol} / \mathrm{l}$ (concentrations that would not trigger automatic exchange transfusion for neonates with ABO HDN in most units); the timing of administration and dose of IVIG vary considerably between studies. Despite these problems, it seems clear that IVIG does confer benefit when administered to neonates with HDN. The published studies suggest that IVIG in this context reduces the number of neonates treated with exchange transfusion, the total length of phototherapy required, and the length of hospital stay. Late anaemia may be more prevalent in those treated with IVIG, presumably because fewer neonates have exchange transfusion and therefore removal of maternal antibody. No major side effects have been reported in the neonates treated with IVIG, but it should be remembered in this context that IVIG is a pooled blood product and so the potential for transmission of blood-borne infections will always be present..$^{51}$

Given these facts, how should neonatal paediatricians approach the use of IVIG in patients with HDN? Clearly, one approach is to wait for results from a definite trial(s) with methodology that reflects modern clinical practice-that is, where the decision to carry out an exchange transfusion is based on the combination of bilirubin concentration, general condition of the neonate, rapid diagnosis of the likely cause of HDN, and response to intensive phototherapy-rather than a didactic approach based solely on bilirubin concentration or rate of rise of bilirubin. This approach is entirely justified given that IVIG can never be guaranteed to be $100 \%$ safe (although blood for exchange transfusion also cannot be guaranteed to be $100 \%$ safe, and, as clinical experience of exchange transfusion itself decreases, this procedure may well have increasing morbidity). Alternatively it may well be justified to electively use IVIG in a small number of selected patients with HDN where the likelihood of exchange transfusion is great - that is, neonates with $\mathrm{Rh}$ disease unmodified by antenatal treatment or neonates with potential ABO HDN where a previous sibling has suffered from severe disease requiring exchange transfusion. As a definitive trial reflecting modern practice recruiting sufficient patients to show a definite reduction in neonates who require exchange transfusion would need to be a large multicentre exercise, the latter option is likely to be followed by those swayed by the current evidence on IVIG for HDN.

\section{CONCLUSIONS}

The diagnosis, acute management, and follow-up of neonates with HDN still represent an important area of activity for maternity/neonatal services. Through the highly successful use of prophylactic $\mathrm{Rh} \mathrm{D}$ immunoglobulin in $\mathrm{Rh} \mathrm{D}$ negative women, Rh D allo-immunisation and its sequelae have greatly diminished. As a result, $\mathrm{ABO}$ incompatibility is now the single largest cause of HDN in the western world. However, with increasing knowledge at the molecular level, and closer liaison between neonatal paediatricians and haematologists, the diagnosis of non-immune causes of HDN is increasing. As these conditions have an inherited basis and therefore have implications for other family members (or future children), it must remain a high priority for all neonatal paediatricians to achieve an accurate diagnosis in all cases of HDN, and emphasises the point that patients with persistent neonatal haemolysis should always be fully investigated and followed up appropriately.

As the efficacy of phototherapy increases, the role of exchange transfusion in the acute management of HDN is rapidly decreasing. This represents a potential management dilemma for neonatal paediatricians, as the vast majority of neonates presenting with hyperbilirubinaemia, and presumed HDN, will respond to phototherapy-based management, thus avoiding any potential hazards associated with exchange transfusion. This makes gauging the appropriate time to intervene with exchange transfusion an increasingly difficult clinical decision. In addition, as the number of exchange transfusions undertaken by most maternity/neonatal services per year is now in single figures, few neonatal trainees can hope to develop practical competence in this technique. In view of these issues, all maternity/neonatal services must develop, maintain and teach appropriate written practice guidelines both for use of exchange transfusion in the management of HDN and for the performance of the procedure itself. Indeed this may be even more important in the future as the use of IVIG and/or metalloporphyrins ${ }^{53}$ further reduces the need for exchange transfusion and continues to change the spectrum of HDN faced by neonatal paediatricians.

Arch Dis Child Fetal Neonatal Ed 2007;92:F83F88.

doi: 10.1136/adc.2005.076794

\section{Authors' affiliations}

N A Murray, Imperial College, Department of Paediatrics, Hammersmith Hospital, London W12 ONN, UK

I A G Roberts, Imperial College Faculty of Medicine, Hammersmith Campus, Hammersmith Hospital, London W12 ONN, UK

Correspondence to: Dr Neil A Murray, Senior Lecturer in Neonatal Medicine, Imperial College, Department of Paediatrics, 5th Floor, Ham House, Hammersmith Hospital, Du Cane Road, London W12 ONN, UK; neil.murray@imperial. ac.uk

Competing interests: None.

\section{REFERENCES}

1 Wang m, Hays T, Ambruso DR, et al. Hemolytic disease of the newborn caused by a high titer antigroup B lgG from a group A mother. Pediatr Blood Cancer 2005:45:861-2.

2 Chen JY, Ling UP. Prediction of the development of neonatal hyperbilirubinemia in $\mathrm{ABO}$ incompatibility. Zhonghua Yi Xue Za Zhi (Taipei) 1994:53:13-18.

3 Oski FA. The erythrocyte and its disorders. In: Nathan A, Oski FA, eds. Hematology of infancy and childhood. Philadelphia: WB Saunders, 1993:18-43.

4 Waldron $P$, de Alarcon P. ABO hemolytic disease of the newborn: a unique constellation of findings in siblings and review of protective mechanisms in the fetal-maternal system. Am J Perinatol 1999:16:391-8.

5 Ziprin JH, Payne E, Hamidi L, et al. ABO incompatibility due to immunoglobulin $G$ anti-B antibodies presenting with severe fetal anaemia. Transfus Med 2005; 15:57-60.

6 Palek J, Jarolim P. Clinical expression and laboratory detection of red blood cell membrane protein mutations. Semin Hematol 1993;30:249-83

7 Delaunay J. Molecular basis of red cell membrane disorders. Acta Haematol 2002; 108:210-18.

8 Tse WT, Lux SE. Red blood cell membrane disorders. Br J Haematol 1999;104:2-13.

9 Luzzatto L. Glucose-6-phosphate dehydrogenase deficiency. In: Nathan A, Oski FA, eds. Hematology of infancy and childhood.4th edn. Philadelphia: WB Saunders, 1993:674-95.

10 Kaplan M. Genetic interactions in the pathogenesis of neonatal hyperbilirubinemia: Gilbert's Syndrome and glucose-6-phosphate dehydrogenase deficiency. J Perinatol 2001;21(Suppl 1):S35-9.

11 Kaplan M, Hammerman C. Glucose-6-phosphate dehydrogenase deficiency: a potential source of severe neonatal hyperbilirubinaemia and kernicterus. Semin Neonatol 2002;7:121-8. 
12 www.rialto.com/favism/english/index.mv (accessed 9 Jan 2007)

13 Gilsanz F, Vega MA, Gomez-Castillo E, et al. Fetal anaemia due to pyruvate kinase deficiency. Arch Dis Child 1993:69:523-4.

14 Zanella A, Bianchi P. Red cell pyruvate kinase deficiency: from genetics to clinical manifestations. Bailliere's Best Pract Res Clin Haematol 2000:13:57-81.

15 Schneider AS. Triosephosphate isomerase deficiency: historical perspectives and molecular aspects. Bailliere's Best Pract Res Clin Haematol 2000;13:119-40.

16 Davies SC, Cronin E, Gill M, et al. Screening for sickle cell disease and thalassaemia: a systematic review with supplementary research. Health Technol Assess. 2000;4: iii-v, 1-99).

17 Higgs DR. Alpha-thalassaemia. Bailliere's Clin Haematol 1993:6:117-50.

18 Chik KW, Shing MM, Li CK, et al. Treatment of hemoglobin Bart's hydrops with bone marrow transplantation. J Pediatr 1998;132:1039-42.

19 Sohan K, Billington M, Pamphilon D, et al. Normal growth and development following in utero diagnosis and treatment of homozygous alphathalassaemia. BJOG 2002;109:1308-10.

20 Lee-Potter JP, Deacon-Smith RA, Simpkiss MJ, et al. A new cause of hemolytic anemia in the newborn. J Clin Pathol 1975;28:317-20.

21 Howard H, Martlew V, McFadyen I, et al. Consequences for fetus and neonate of maternal red cell alloimmunisation. Arch Dis Child Fetal Neonatal Ed 1998;78:F62-6.

22 Stockman JA, de Alarcon PA. Overview of the state of the art of Rh disease: history, current clinical management, and recent progress. J Pediatr Hematol Oncol 2001;23:385-93.

23 Thompson J. Haemolytic disease of the newborn: the new NICE guidelines. J Fam Health Care 2002;12:133-6.

24 Vaughan Jl, Manning M, Warwick RM, et al. Inhibition of erythroid progenitor cells by anti-Kell antibodies in fetal alloimmune anemia. NEngl J Med 1998;338:798-803

25 Bennett PR, Le Van Kim C, Colin Y, et al. Prenatal determination of fetal RhD type by DNA amplification. N Engl J Med, 1993 26, 329:607-10.

26 Al-Mufti R, Howard C, Overton T, et al. Detection of fetal messenger ribonucleic acid in maternal blood to determine fetal RhD status as a strategy for noninvasive prenatal diagnosis. Am J Obstet Gynecol 1998;179:210-4.

27 Bianchi DW, Avent ND, Costa JM, et al. Noninvasive prenatal diagnosis of fetal Rhesus D: ready for Prime(r) Time. Obstet Gynecol 2005; 106:841-4.

28 Urbaniak SJ, Greiss MA. RhD haemolytic disease of the fetus and the newborn. Blood Rev 2000;14:44-61.

29 Harkness UF, Spinnato JA. Prevention and management of $\mathrm{RhD}$ isoimmunization. Clin Perinatol 2004:31:721-42.

30 Yaseen H, Khalaf M, Rashid N, et al. Does prophylactic phototherapy prevent hyperbilirubinemia in neonates with $A B O$ incompatibility and positive Coombs' test? J Perinatol 2005;25:590-4.

31 Newman TB, Liljestrand, PJeremy RJ, et al. Outcomes among newborns with total serum bilirubin levels of $25 \mathrm{mg}$ per deciliter or more. N Engl J Med, 2006;4, 354:1889-900.

32 van Kamp IL, Klumper FJ, Meerman $\mathrm{RH}_{\text {, et al. }}$ Treatment of fetal anemia due to red-cell alloimmunization with intrauterine transfusions in the Netherlands, 1988-1999. Acta Obstet Gynecol Scand 2004;83:731-7.

33 Murray NA, Roberts IA. Neonatal transfusion practice. Arch Dis Child Fetal Neonatal Ed 2004;89:F101-7.

34 Dinesh D. Review of positive direct antiglobulin tests found on cord blood sampling. J Paediatr Child Health 2005;41:504-7.

35 Cortey A, Brossard Y. [Adverse effects and patien information]. J Gynecol Obstet Biol Reprod (Paris) 2006;35(1 Suppl):1S112-1S118.

36 Cremer RJ, Perryman PW, Richards DH. Influence of light on the hyperbilirubinaemia of infants. Lancet, 1958 24, 1(7030):1094-7.

37 Vreman HJ, Wong RJ, Stevenson DK. Phototherapy: current methods and future directions. Semin Perinatol 2004;28:326-33.

38 Hart G, Cameron R. The importance of irradiance and area in neonatal phototherapy. Arch Dis Child Fetal Neonatal Ed 2005;90:F437-40.

39 Vreman HJ, Wong RJ, Stevenson DK, et al. Lightemitting diodes: a novel light source for phototherapy. Pediatr Res 1998;44:804-9.

40 Seidman DS, Moise J, Ergaz Z, et al. A prospective randomized controlled study of phototherapy using blue and blue-green light-emitting devices, and conventional halogen-quartz phototherapy. J Perinatol 2003;23:123-7.

41 Thaithumyanon P, Visutiratmanee C. Double phototherapy in jaundiced term infants with hemolysis. J Med Assoc Thai 2002;85:1176-81.

42 McDonagh AF. Phototherapy: from ancient Egypt to the new millennium. J Perinatol 2001;21(Suppl 1):S7-S12.

43 Miqdad AM, Abdelbasit OB, Shaheed MM, et al. Intravenous immunoglobulin G (IVIG) therapy for significant hyperbilirubinemia in $A B O$ hemolytic disease of the newborn. J Matern Fetal Neonatal Med 2004; 16:163-6.

44 Mukhopadhyay K, Murki S, Narang A, et al. Intravenous immunoglobulins in rhesus hemolytic disease. Indian J Pediatr 2003;70:697-9.

45 Alpay F, Sarici SU, Okutan V, et al. High-dose intravenous immunoglobulin therapy in neonatal immune haemolytic jaundice. Acta Paediatr 1999;88:216-9.

46 Dagoglu T, Ovali F, Samanci N, et al. High-dose intravenous immunoglobulin therapy for rhesus haemolytic disease. J Int Med Res 1995;23:264-71.

47 Voto LS, Sexer H, Ferreiro G, et al. Neonatal administration of high-dose intravenous immunoglobulin in rhesus hemolytic disease. J Perinat Med 1995;23:443-51.

48 Rubo J, Albrecht K, Lasch P, et al. High-dose intravenous immune globulin therapy for hyperbilirubinemia caused by $\mathrm{Rh}$ hemolytic disease $J$ Pediatr 1992;121:93-7.

49 Alcock GS, Liley H. Immunoglobulin infusion for isoimmune haemolytic jaundice in neonates. Cochrane Database Syst Rev 2002;3:CD003313.

50 Gottstein R, Cooke RW. Systematic review of intravenous immunoglobulin in haemolytic disease of the newborn. Arch Dis Child Fetal Neonatal Ed 2003;88:F6-10.

51 Hayakawa F, Imada K, Towatari M, et al. Lifethreatening human parvovirus B19 infection transmitted by intravenous immune globulin. Br J Haematol 2002;1 18:1187-9.

52 Quinti I, Pierdominici M, Marziali M, et al. European surveillance of immunoglobulin safety-results of initial survey of 1243 patients with primary immunodeficiencies in 16 countries. Clin Immunol 2002;104:231-6.

53 Dennery PA. Metalloporphyrins for the treatment of neonatal jaundice. Curr Opin Pediatr 2005;17:167-9.

\section{The management of preterm labour}

\section{Jayanta Chatterjee, Joanna Gullam, Manu Vatish, Steve Thornton}

$P$ reterm labour is the onset of regular uterine contractions associated with progressive cervical change between viability and 37 completed weeks of gestation. The incidence is between 5\% and $10 \%$ in most developed nations. In the US, the incidence has increased from $9 \%$ to $12 \%$ in the past two decades. Preterm delivery can be associated with immediate and long-term neonatal complications. Long-term morbidity includes cerebral palsy, neurodevelopmental delay and chronic lung disease. The neonatal outcome is dependent on the gestational age at delivery and associated features such as infection. The lower the gestational age, the higher the risk of mortality and morbidity. The management of preterm labour involves identification of high-risk women, prevention and treatment.

\section{IDENTIFICATION OF AND PREVENTION IN WOMEN AT RISK}

The identification of women at high risk of preterm delivery remains a major challenge. Scoring systems based on socioeconomic status, obstetric or medical history and antenatal events in the index pregnancy have shown a suboptimal correlation with subsequent preterm birth. ${ }^{1}$ This is primarily because the single greatest risk factor is a history of preterm labour, so delivery cannot be reliably predicted in the first pregnancy. The risk of preterm delivery after one and two previous preterm deliveries has been given as $15 \%$ and $41 \%$, respectively ${ }^{1}$; however, such figures are difficult to apply to individuals as the risk is dependent on the cause and the gestational age of the previous preterm delivery.

Investigations such as fetal fibronectin or cervical ultrasound can be used to identify women at high risk. A positive swab for vaginal fetal fibronectin taken in the late second or early third trimester increases the likelihood of delivery before 34 weeks by a factor of $4 .^{2}$ Likewise, a negative swab reduces the likelihood of delivery to 0.78 . Such results from 